\title{
HSC/10/04
}

\section{Ruin Probability in Finite Time}

\section{Krzysztof Burnecki* Marek Teuerle*}

* Hugo Steinhaus Center, Wrocław University of Technology, Poland

Hugo Steinhaus Center Wrocław University of Technology

Wyb. Wyspiańskiego 27, 50-370 Wrocław, Poland http://www.im.pwr.wroc.pl/ hugo/ 


\title{
Ruin Probability in Finite Time ${ }^{1}$
}

\author{
Krzysztof Burnecki ${ }^{2}$ and Marek Teuerle ${ }^{2}$
}

\begin{abstract}
The ruin probability in finite time can only be calculated analytically for a few special cases of the claim amount distribution. The most classic example is discussed in Section 1.2. The value can always be computed directly using Monte Carlo simulations, however, this is usually a time-consuming procedure. Thus, finding a reliable approximation is really important from a practical point of view. The most important approximations of the finite time ruin probability are presented in Section 1.3. They are further illustrated in Section 1.4 using the Danish fire losses dataset, which concerns major fire losses in profits that occurred between 1980 and 2002 and were recorded by Copenhagen Re.
\end{abstract}

Keywords: Insurance risk model; Ruin probability; Segerdahl approximation; De Vylder approximation; Diffusion approximation; Brownian motion; Levy motion

JEL: C15, C46, C63, G22, G32

\footnotetext{
${ }^{1}$ Chapter prepared for the $2^{\text {nd }}$ edition of Statistical Tools for Finance and Insurance, P.Cizek, W.Härdle, R.Weron (eds.), Springer-Verlag, forthcoming in 2011.

${ }^{2}$ Hugo Steinhaus Center, Wrocław University of Technology, Poland
} 


\title{
1 Ruin probability in finite time
}

\author{
Krzysztof Burnecki and Marek Teuerle
}

\subsection{Introduction}

In examining the nature of the risk associated with a portfolio of business, it is often of interest to assess how the portfolio may be expected to perform over an extended period of time. One approach involves the use of ruin theory (Panjer and Willmot, 1992). Ruin theory is concerned with the excess of the income (with respect to a portfolio of business) over the outgo, or claims paid. This quantity, referred to as insurer's surplus, varies in time. Specifically, ruin is said to occur if the insurer's surplus reaches a specified lower bound, e.g. minus the initial capital. One measure of risk is the probability of such an event, clearly reflecting the volatility inherent in the business. In addition, it can serve as a useful tool in long range planning for the use of insurer's funds.

We recall from Chapter ?? that the classical risk process $\left\{R_{t}\right\}_{t \geq 0}$ is given by

$$
R_{t}=u+c t-\sum_{i=1}^{N_{t}} X_{i},
$$

where the initial capital of the insurance company is denoted by $u$, the homogeneous Poisson process (HPP) $N_{t}$ with intensity (rate) $\lambda$ describes the number of claims in $(0, t]$ interval and claim severities are random, given by i.i.d. nonnegative sequence $\left\{X_{k}\right\}_{k=1}^{\infty}$ with mean value $\mu$ and variance $\sigma^{2}$, independent of $N_{t}$. The insurance company receives a premium at a constant rate $c$ per unit time, where $c=(1+\theta) \lambda \mu$ and $\theta>0$ is called the relative safety loading.

We define the claim surplus process $\left\{S_{t}\right\}_{t \geq 0}$ as

$$
S_{t}=u-R_{t}=\sum_{i=1}^{N_{t}} X_{i}-c t
$$


and the time to ruin as $\tau(u)=\inf \left\{t \geq 0: R_{t}<0\right\}=\inf \left\{t \geq 0: S_{t}>u\right\}$. Let $L=\sup _{0 \leq t<\infty}\left\{S_{t}\right\}$ and $L_{T}=\sup _{0 \leq t<T}\left\{S_{t}\right\}$. The ruin probability in infinite time, i.e. the probability that the capital of an insurance company ever drops below zero can be then written as

$$
\psi(u)=\mathrm{P}(\tau(u)<\infty)=\mathrm{P}(L>u) .
$$

We note that the above definition implies that the relative safety loading $\theta$ has to be positive, otherwise $c$ would be less than $\lambda \mu$ and thus with probability 1 the risk business would become negative in infinite time. The ruin probability in finite time $T$ is given by

$$
\psi(u, T)=\mathrm{P}(\tau(u) \leq T)=\mathrm{P}\left(L_{T}>u\right) .
$$

From a practical point of view, $\psi(u, T)$, where $T$ is related to the planning horizon of the company, is regarded as more interesting than $\psi(u)$. Most insurance managers will closely follow the development of the risk business and increase the premium if the risk business behaves badly. The planning horizon may be thought of as the sum of the following: the time until the risk business is found to behave "badly", the time until the management reacts and the time until a decision of a premium increase takes effect. Therefore, in non-life insurance, it may be natural to regard $T$ equal to four or five years as reasonable (Grandell, 1991).

Let us now analyze the finite-time ruin probability for the probably most practical generalization of the classical risk process, where the occurrence of the claims is described by the non-homogeneous Poisson process (NHPP), see Chapter ??. It can be proved that switching from HPP to NHPP results only in altering the time horizon $T$. This stems from the following fact. Consider a risk process $\tilde{R}_{t}$ driven by a non-homogeneous Poisson process $\tilde{N}_{t}$ with the intensity function $\lambda(t)$, namely

$$
\tilde{R}_{t}=u+(1+\theta) \mu \int_{0}^{t} \lambda(s) d s-\sum_{i=1}^{\tilde{N}_{t}} X_{i} .
$$

Define now $\Lambda_{t}=\int_{0}^{t} \lambda(s) d s$ and $R_{t}=\tilde{R}\left(\Lambda_{t}^{-1}\right)$. Then the counting process $N_{t}=\tilde{N}\left(\Lambda_{t}^{-1}\right)$ is a standard Poisson process, and therefore,

$$
\tilde{\psi}(u, T)=\mathrm{P}\left\{\inf _{0<t \leq T}\left(\tilde{R}_{t}\right)<0\right\}=\mathrm{P}\left\{\inf _{0<t \leq \Lambda_{T}}\left(R_{t}\right)<0\right\}=\psi\left(u, \Lambda_{T}\right) .
$$


The time scale defined by $\Lambda_{t}^{-1}$ is called the operational time scale.

The ruin probability in finite time can only be calculated analytically for a few special cases of the claim amount distribution. The most classic example is discussed in Section 1.2. The value of $\psi(u, T)$ can always be computed directly using Monte Carlo simulations, however, this is usually a time-consuming procedure. Thus, finding a reliable approximation is really important from a practical point of view. The most important approximations of the finite time ruin probability are presented in Section 1.3. They are further illustrated in Section 1.4 using the Danish fire losses dataset, introduced in Chapter ??, which concerns major fire losses in profits that occurred between 1980 and 2002 and were recorded by Copenhagen Re.

We note that ruin theory has been also recently employed as an interesting tool in operational risk (Degen, Embrechts, and Lambrigger, 2007; Kaishev, Dimitrova, and Ignatov, 2008). In the view of the data already available on operational risk, ruin type estimates may become useful (Embrechts, Kaufmann, and Samorodnitsky, 2004).

\subsubsection{Light- and heavy-tailed distributions}

A distribution $F_{X}(x)$ is said to be light-tailed, if there exist constants $a>0$, $b>0$ such that $\bar{F}_{X}(x)=1-F_{X}(x) \leq a e^{-b x}$ or, equivalently, if there exist $z>0$, such that $M_{X}(z)<\infty$, where $M_{X}(z)$ is the moment generating function, see Chapter ??. Distribution $F_{X}(x)$ is said to be heavy-tailed, if for all $a>0$, $b>0 \bar{F}_{X}(x)>a e^{-b x}$, or, equivalently, if $\forall z>0 M_{X}(z)=\infty$. We study here eight claim size distributions, as listed in Table 1.1

In the case of light-tailed claims the adjustment coefficient (also called the Lundberg exponent) plays a key role in calculating the ruin probability. Let $\gamma=\sup _{z}\left\{M_{X}(z)\right\}<\infty$ and let $R$ be a positive solution of the equation:

$$
1+(1+\theta) \mu R=M_{X}(R), \quad R<\gamma
$$

If there exists a non-zero solution $R$ to the above equation, we call it an adjustment coefficient. Clearly, $R=0$ satisfies equation (1.5), but there may exist a positive solution as well (this requires that $X$ has a moment generating function, thus excluding distributions such as Pareto and the log-normal). To see the plausibility of this result, note that $M_{X}(0)=1, M_{X}^{\prime}(z)<0, M_{X}^{\prime \prime}(z)>0$ and $M_{X}^{\prime}(0)=-\mu$. Hence, the curves $y=M_{X}(z)$ and $y=1+(1+\theta) \mu z$ may intersect, as shown in Figure 1.1. 
Table 1.1: Typical claim size distributions. In all cases $x \geq 0$.

\begin{tabular}{lll}
\hline \hline \multicolumn{1}{c}{ Name } & Parameters & pdf \\
\hline Exponential & $\beta>0$ & \multicolumn{2}{c}{ Light-tailed distributions } \\
Gamma & $\alpha>0, \beta>0$ & $f_{X}(x)=\beta \exp (-\beta x)$ \\
Weibull & $\beta>0, \tau \geq 1$ & $f_{X}(x)=\frac{\beta^{\alpha}}{\Gamma(\alpha)} x^{\alpha-1} \exp (-\beta x)$ \\
Mixed exp's & $\beta_{i}>0, \sum_{i=1}^{n} a_{i}=1$ & $f_{X}(x)=\sum_{i=1}^{n}\left\{a_{i} \beta_{i} \exp \left(-\beta_{i} x\right)\right\}$ \\
\hline \multicolumn{3}{c}{ Heavy-tailed distributions } \\
\hline Weibull & $\beta>0,0<\tau<1$ & $f_{X}(x)=\beta \tau x^{\tau-1} \exp \left(-\beta x^{\tau}\right)$ \\
Log-normal & $\mu \in \mathbb{R}, \sigma>0$ & $f_{X}(x)=\frac{1}{\sqrt{2 \pi} \sigma x} \exp \left\{-\frac{(\ln x-\mu)^{2}}{2 \sigma^{2}}\right\}$ \\
Pareto & $\alpha>0, \lambda>0$ & $f_{X}(x)=\frac{\alpha}{\lambda+x}\left(\frac{\lambda}{\lambda+x}\right)^{\alpha}$ \\
Burr & $\alpha>0, \lambda>0, \tau>0$ & $f_{X}(x)=\frac{\alpha \tau \lambda^{\alpha} x^{\tau-1}}{\left(\lambda+x^{\tau}\right)^{\alpha+1}}$ \\
\hline \hline
\end{tabular}

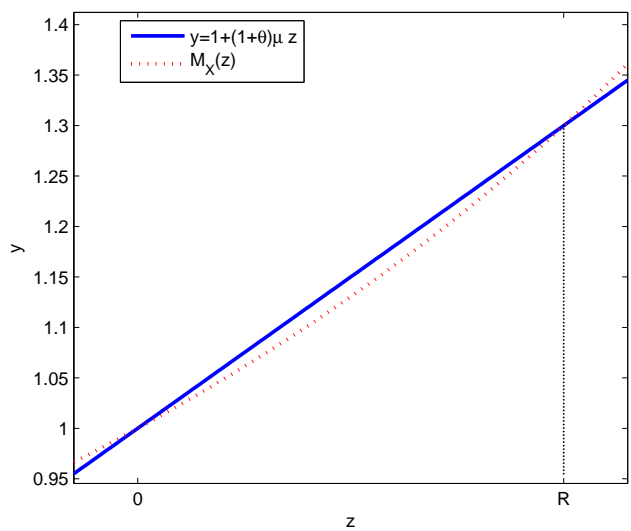

Figure 1.1: Illustration of the existence of the adjustment coefficient. 
An analytical solution to equation (1.5) exists only for few claim distributions. However, it is quite easy to obtain a numerical solution. The coefficient $R$ satisfies the inequality:

$$
R<\frac{2 \theta \mu}{\mu^{(2)}}
$$

where $\mu^{(2)}=\mathrm{E}\left(X_{i}^{2}\right)$, see Asmussen (2000). Let $D(z)=1+(1+\theta) \mu z-M_{X}(z)$. Thus, the adjustment coefficient $R>0$ satisfies the equation $D(R)=0$. In order to get the solution one may use the Newton-Raphson formula

$$
R_{j+1}=R_{j}-\frac{D\left(R_{j}\right)}{D^{\prime}\left(R_{j}\right)},
$$

with the initial condition $R_{0}=2 \theta \mu / \mu^{(2)}$, where $D^{\prime}(z)=(1+\theta) \mu-M_{X}^{\prime}(z)$. Moreover, if it is possible to calculate the third raw moment $\mu^{(3)}$, we can obtain a sharper bound than (1.6) (Panjer and Willmot, 1992):

$$
R<\frac{12 \mu \theta}{3 \mu^{(2)}+\sqrt{9\left(\mu^{(2)}\right)^{2}+24 \mu \mu^{(3)} \theta}}
$$

and use it as the initial condition in (1.7).

\subsection{Exact ruin probabilities in finite time}

We are now interested in the probability that the insurer's capital as defined by (1.1) remains non-negative for a finite period $T$. We assume that the claim counting process $N_{t}$ is a homogeneous Poisson process (HPP) with rate $\lambda$, and consequently, the total claims (aggregate loss) process is a compound Poisson process. Premiums are payable at rate $c$ per unit time. We recall that if $N_{t}$ is a non-homogeneous Poisson process, which will be the case for all numerical examples, then it is enough to rescale the time horizon $T$ for the ruin probability obtained for the classic HPP case, see the discussion at the end of Section 1.1.

In contrast to the infinite time case there is no general result for the ruin probability like the Pollaczek-Khinchin formula (Burnecki, Miśta, and Weron, 2005). In the literature one can only find a partial integro-differential equation which satisfies the probability of non-ruin, see Panjer and Willmot (1992). An explicit result is merely known for the exponential claims, and even is this case numerical integration is needed (Asmussen, 2000). 


\subsubsection{Exponential claim amounts}

First, in order to simplify the formulae, let us assume that claims have the exponential distribution with $\beta=1$ and the amount of premium is $c=1$. Then

$$
\psi(u, T)=\lambda \exp \{-(1-\lambda) u\}-\frac{1}{\pi} \int_{0}^{\pi} \frac{f_{1}(x) f_{2}(x)}{f_{3}(x)} d x
$$

where

$$
\begin{gathered}
f_{1}(x)=\lambda \exp \{2 \sqrt{\lambda} T \cos x-(1+\lambda) T+u(\sqrt{\lambda} \cos x-1)\}, \\
f_{2}(x)=\cos (u \sqrt{\lambda} \sin x)-\cos (u \sqrt{\lambda} \sin x+2 x),
\end{gathered}
$$

and

$$
f_{3}(x)=1+\lambda-2 \sqrt{\lambda} \cos x
$$

Now, notice that the case $\beta \neq 1$ is easily reduced to $\beta=1$, using the formula:

$$
\psi_{\lambda, \beta}(u, T)=\psi_{\frac{\lambda}{\beta}, 1}(\beta u, \beta T) .
$$

Moreover, the assumption $c=1$ is not restrictive since we have

$$
\psi_{\lambda, c}(u, T)=\psi_{\lambda / c, 1}(u, c T) .
$$

Table 1.2 shows the exact values of the ruin probability for a NHPP with the intensity rate $\lambda(t)=17.9937+7.1518 t$ and exponential claims with $\beta=$ $1.9114 \cdot 10^{-6}$ (the parameters were estimated in Chapter ?? for the Danish fire losses dataset) with respect to the initial capital $u$ and the time horizon $T$. The relative safety loading $\theta$ is set to $30 \%$.

\subsection{Approximations of the ruin probability in finite time}

In this section, we present six different approximations. We illustrate them on a common example, namely a NHPP with the intensity rate $\lambda(t)=17.9937+$ $7.1518 t$, the mixture of two exponentials claims with $\beta_{1}=3.8617 \cdot 10^{-7}, \beta_{2}=$ $3.6909 \cdot 10^{-6}$ and $a=0.2568$ (the parameters were estimated in Chapter ?? 
Table 1.2: The ruin probability for a NHPP with the intensity function $\lambda(t)=$ $17.9937+7.1518 t$, exponential claims with $\beta=1.9114 \cdot 10^{-6}$ and $\theta=0.3$ ( $u$ in DKK millions).

\begin{tabular}{lrrrrrr}
\hline \hline$u$ & 0 & 1 & 2 & 5 & 10 & 15 \\
\hline$\psi(u, 1)$ & 0.745163 & 0.447479 & 0.263129 & 0.046887 & 0.001719 & 0.000039 \\
$\psi(u, 2)$ & 0.763315 & 0.482797 & 0.303608 & 0.072409 & 0.005595 & 0.000338 \\
$\psi(u, 3)$ & 0.767620 & 0.491540 & 0.314220 & 0.080994 & 0.007930 & 0.000694 \\
$\psi(u, 4)$ & 0.768802 & 0.493980 & 0.317247 & 0.083702 & 0.008891 & 0.000903 \\
$\psi(u, 5)$ & 0.769230 & 0.494649 & 0.318087 & 0.084768 & 0.009341 & 0.001029 \\
\hline \hline
\end{tabular}

STF2ruin02.m

for the Danish fire losses dataset) and the relative safety loading $\theta=30 \%$. Numerical comparison of the approximations is given in Section 1.4. All the formulas presented in this section assume the classic form of the risk process, however, we recall that if the process $N_{t}$ is a NHPP, then it is enough to rescale the time horizon $T$ for the ruin probability obtained for the classic HPP case.

\subsubsection{Monte Carlo method}

The ruin probability in finite time can always be approximated by means of Monte Carlo simulations. Table 1.3 shows the output for the with respect to the initial capital $u$ and the time horizon $T$. We note that the Monte Carlo method will be used as a reference method when comparing different finite time approximations in Section 1.4 .

\subsubsection{Segerdahl normal approximation}

The following result due to Segerdahl (1955) is said to be a time-dependent version of the Cramér-Lundberg approximation .

$$
\psi_{S}(u, T)=C \exp (-R u) \Phi\left(\frac{T-u m_{L}}{\omega_{L} \sqrt{u}}\right),
$$


Table 1.3: Monte Carlo results (50 x 10000 simulations) for a NHPP with the intensity function $\lambda(t)=17.9937+7.1518 t$, mixture of two exponentials claims with $\beta_{1}=3.8617 \cdot 10^{-7}, \beta_{2}=3.6909 \cdot 10^{-6}, a=0.2568$ and $\theta=0.3$ ( $u$ in DKK million).

\begin{tabular}{lrrrrrr}
\hline \hline$u$ & 0 & 1 & 5 & 10 & 20 & 50 \\
\hline$\psi(u, 1)$ & 0.702560 & 0.561842 & 0.302862 & 0.130702 & 0.020922 & 0.000062 \\
$\psi(u, 2)$ & 0.746522 & 0.623986 & 0.369984 & 0.193242 & 0.046346 & 0.000424 \\
$\psi(u, 3)$ & 0.758588 & 0.638384 & 0.403508 & 0.224844 & 0.064726 & 0.001566 \\
$\psi(u, 4)$ & 0.762924 & 0.646102 & 0.417342 & 0.238882 & 0.075002 & 0.001900 \\
$\psi(u, 5)$ & 0.763706 & 0.653820 & 0.419522 & 0.244244 & 0.081800 & 0.002300 \\
\hline \hline
\end{tabular}

STF2ruin03.m

Table 1.4: The Segerdahl approximation for the same parameters as in Table 1.3.

\begin{tabular}{lrrrrrr}
\hline \hline$u$ & 0 & 1 & 5 & 10 & 20 & 50 \\
\hline$\psi(u, 1)$ & 0.727958 & 0.593121 & 0.253626 & 0.110960 & 0.025333 & 0.000418 \\
$\psi(u, 2)$ & 0.727958 & 0.654281 & 0.425585 & 0.237081 & 0.065324 & 0.001218 \\
$\psi(u, 3)$ & 0.727958 & 0.654281 & 0.426969 & 0.250430 & 0.086090 & 0.003089 \\
$\psi(u, 4)$ & 0.727958 & 0.654281 & 0.426969 & 0.250430 & 0.086152 & 0.003507 \\
$\psi(u, 5)$ & 0.727958 & 0.654281 & 0.426969 & 0.250430 & 0.086152 & 0.003508 \\
\hline \hline
\end{tabular}

STF2ruin04.m

where $C=\theta \mu /\left\{M_{X}^{\prime}(R)-\mu(1+\theta)\right\}, \quad m_{L}=C\left\{\lambda M_{X}^{\prime}(R)-1\right\}^{-1}$ and $\omega_{L}^{2}=\lambda M_{X}^{\prime \prime}(R) m_{L}^{3}$. This method requires existence of the adjustment coefficient, so the moment generating function. This implies that only light-tailed distributions can be used. Numerical evidence shows that the Segerdahl approximation gives the best results for large values of the initial capital $u$, see Asmussen (2000).

In Table 1.4 the results of the Segerdahl approximation are presented with respect to the initial capital $u$ and the time horizon $T$. We can see that the 
Table 1.5: The diffusion approximation by Brownian motion for the same parameters as in Table 1.3

\begin{tabular}{lrrrrrr}
\hline \hline$u$ & 0 & 1 & 5 & 10 & 20 & 50 \\
\hline$\psi(u, 1)$ & 1.000000 & 0.830499 & 0.366727 & 0.108608 & 0.004407 & 0.000000 \\
$\psi(u, 2)$ & 1.000000 & 0.860162 & 0.467565 & 0.213980 & 0.040964 & 0.000097 \\
$\psi(u, 3)$ & 1.000000 & 0.863616 & 0.480142 & 0.239134 & 0.052420 & 0.000531 \\
$\psi(u, 4)$ & 1.000000 & 0.863892 & 0.481159 & 0.231498 & 0.053569 & 0.000657 \\
$\psi(u, 5)$ & 1.000000 & 0.863902 & 0.481196 & 0.231549 & 0.053615 & 0.000665 \\
\hline \hline
\end{tabular}

STF2ruin05.m

approximation in the considered case yields quite accurate results for larger $u$ 's except for $u=50$ million DKK, see Table 1.3, but this effect can be explained by very small values of the probability.

\subsubsection{Diffusion approximation by Brownian motion}

The idea of a diffusion (weak) approximation goes back to Iglehart (1969). The first approximation we study assumes that the distribution of claim sizes belongs to the domain of attraction of the normal law, i.e. claims are i.i.d. and have light tails. This leads to the approximation of the risk process by a diffusion driven by the Brownian motion. In this case one can calculate an analytical approximation formula for the ruin probability in finite time (Grandell, 1991):

$$
\begin{aligned}
\psi_{D B}(u, t) & =P\left\{\inf _{0 \leq t \leq T}\left(u+(c-\lambda \mu) t-\sqrt{\lambda \mu^{(2)}} B(t)\right)<0\right\}= \\
& =I G\left(\frac{T \mu_{c}^{2}}{\sigma_{c}^{2}} ;-1 ; \frac{u\left|\mu_{c}\right|}{\sigma_{c}^{2}}\right),
\end{aligned}
$$

where $B(t)$ denotes the Brownian motion, $\mu_{c}=\lambda \theta \mu, \sigma_{c}=\lambda \mu^{(2)}$ and $I G(\cdot ; \zeta ; u)$ denotes the inverse Gaussian distribution function, namely

$$
I G(x ; \zeta ; u)=1-\Phi(u / \sqrt{x}-\zeta \sqrt{x})+\exp (2 \zeta u) \Phi(-u / \sqrt{x}-\zeta \sqrt{x}) .
$$

This formula can be also obtained by matching the first two moments of the claim surplus process $S_{t}$ and a Brownian motion with drift (arithmetic Brow- 
nian motion), and noting that such an approximation implies that the first passage probabilities are close. The first passage probability serves as the ruin probability, see Asmussen (2000). We also note that in order to apply this approximation we need the existence of the second moment of the claim size distribution.

Table 1.5 shows the results of the diffusion approximation by Brownian motion with respect to the initial capital $u$ and the time horizon $T$. The results lead to the conclusion that the approximation does not produce accurate results for such a choice of the claim size distribution. Only when $u=10$ million DKK the results are acceptable, compare with the reference values in Table 1.3

\subsubsection{Corrected diffusion approximation}

The idea presented in Section 1.3.3 ignores the presence of jumps in the risk process (the Brownian motion with drift can continuous trajectories). The corrected diffusion approximation takes this and other deficits into consideration (Asmussen, 2000). Under the assumption that $c=1$, see relation (1.13), we have

$$
\psi_{C D}(u, t)=I G\left(\frac{T \delta_{1}}{u^{2}}+\frac{\delta_{2}}{u} ;-\frac{R u}{2} ; 1+\frac{\delta_{2}}{u}\right),
$$

where $R$ is the adjustment coefficient, $\delta_{1}=\lambda M_{X}^{\prime \prime}\left(\gamma_{0}\right), \delta_{2}=M_{X}^{\prime \prime \prime}\left(\gamma_{0}\right) /\left\{3 M_{X}^{\prime \prime}\left(\gamma_{0}\right)\right\}$ and $\gamma_{0}$ satisfies the equation: $\kappa^{\prime}\left(\gamma_{0}\right)=0$, where $\kappa(s)=\lambda\left\{M_{X}(s)-1\right\}-s$. Similarly as in the Segerdahl approximation, the method requires existence of the moment generating function, so we can use it only for light-tailed distributions.

In Table 1.6 the results of the corrected diffusion approximation are given with respect to the initial capital $u$ and the time horizon $T$. It turns out that corrected diffusion method gives surprisingly good results and is vastly superior to the ordinary diffusion approximation, compare with the reference values in Table 1.3

\subsubsection{Diffusion approximation by $\alpha$-stable Lévy motion}

In this section we present the $\alpha$-stable Lévy motion approximation introduced by Furrer, Michna, and Weron (1997). This is an extension of the Brownian motion approximation approach. It can be applied when claims are heavy-tailed (with power-law tails), which, as the empirical results presented in Chapter ?? show, is statistically justified. 
Table 1.6: The corrected diffusion approximation for the same parameters as in Table 1.3

\begin{tabular}{lrrrrrr}
\hline \hline$u$ & 0 & 1 & 5 & 10 & 20 & 50 \\
\hline$\psi(u, 1)$ & 0.650009 & 0.559629 & 0.299801 & 0.130689 & 0.021822 & 0.000005 \\
$\psi(u, 2)$ & 0.716917 & 0.640833 & 0.407336 & 0.228746 & 0.069354 & 0.001368 \\
$\psi(u, 3)$ & 0.726023 & 0.652191 & 0.424489 & 0.247849 & 0.084042 & 0.003061 \\
$\psi(u, 4)$ & 0.726957 & 0.653363 & 0.426310 & 0.249983 & 0.085927 & 0.003471 \\
$\psi(u, 5)$ & 0.727007 & 0.653426 & 0.426410 & 0.250100 & 0.086037 & 0.003502 \\
\hline \hline
\end{tabular}

We assume that the distribution of the claim sizes belongs to the domain of attraction of the stable law, that is:

$$
\frac{1}{\varphi(n)} \sum_{k=1}^{n}\left(X_{k}-\mu\right) \stackrel{d}{\rightarrow} Z_{\alpha, \beta}(1)
$$

where $\varphi(n)=L(n) n^{1 / \alpha}$ for $L(n)$ being a slowly varying function in infinity, $Z_{\alpha, \beta}(t)$ is the $\alpha$-stable Lévy motion with scale parameter 1 , skewness parameter $\beta$, and $1<\alpha<2$ (Nolan, 2010).

If $\varphi(n)=\sigma^{\prime} n^{1 / \alpha}$, so that $\left\{X_{k}: k \in \mathbb{N}\right\}$ belong to the so-called normal domain of attraction, then the diffusion approximation with $\alpha$-stable Lévy motion is given by:

$$
\psi_{D S}(u, T)=P\left(\inf _{0 \leq t \leq T}\left(u+(c-\lambda \mu) t-\sigma^{\prime} \lambda^{1 / \alpha} Z_{\alpha, \beta}(t)\right)<0\right),
$$

where this probability can be calculated via Monte Carlo method by simulating trajectories of $\alpha$-stable Lévy motion.

In order to illustrate formula (1.19) we cannot consider mixture of exponentials case which was discussed in Sections 1.3.1 1.3.4 as it belongs to the domain of attraction of normal law. Let us now assume that the claim amounts are Pareto distributed with parameters $1<\alpha^{\prime}<2$ and $\lambda^{\prime}$. One may check (Nolan, 2010) that the Pareto distribution belongs to the domain of attraction of $\alpha$-stable law with

$$
\alpha=\alpha^{\prime}
$$


Table 1.7: Diffusion approximation with $\alpha$-stable Lévy motion for a NHPP with the intensity function $\lambda(t)=17.9937+7.1518 t$, Pareto claims with $\alpha^{\prime}=1.3127, \lambda^{\prime}=4.0588 \cdot 10^{5}$ and $\theta=0.3$ ( $u$ in DKK million).

\begin{tabular}{lrrrrrr}
\hline \hline$u$ & 0 & 1 & 5 & 10 & 20 & 50 \\
\hline$\psi(u, 1)$ & 0.469314 & 0.383986 & 0.232228 & 0.154332 & 0.087890 & 0.033532 \\
$\psi(u, 2)$ & 0.563410 & 0.491590 & 0.357850 & 0.280278 & 0.200526 & 0.106646 \\
$\psi(u, 3)$ & 0.605470 & 0.540044 & 0.417866 & 0.345702 & 0.268440 & 0.168546 \\
$\psi(u, 4)$ & 0.629782 & 0.568426 & 0.453638 & 0.385306 & 0.311520 & 0.212744 \\
$\psi(u, 5)$ & 0.646558 & 0.587962 & 0.478302 & 0.412866 & 0.341622 & 0.245320 \\
\hline \hline
\end{tabular}

STF2ruin07.m

and

$$
\sigma^{\prime}=\lambda^{\prime}\left(\frac{\pi}{2 \Gamma\left(\alpha^{\prime}\right) \sin \left(\frac{\alpha^{\prime} \pi}{2}\right)}\right)^{1 / \alpha^{\prime}}=\lambda^{\prime}\left(\frac{\Gamma\left(2-\alpha^{\prime}\right)}{\alpha^{\prime}-1}\left|\cos \frac{\pi \alpha^{\prime}}{2}\right|\right)^{1 / \alpha^{\prime}}
$$

Table 1.7 depicts the results of the diffusion approximation with $\alpha$-stable Lévy motion for a NHPP with the intensity rate $\lambda(t)$, Pareto claims with $\alpha^{\prime}, \lambda^{\prime}$ with respect to the initial capital $u$ and the time horizon $T$. The relative safety loading $\theta$ equals $30 \%$.

\subsubsection{Finite time De Vylder approximation}

Let us recall the idea of the De Vylder approximation in infinite time (Burnecki, Miśta, and Weron, 2005): we replace the claim surplus process with the one with $\theta=\bar{\theta}, \lambda=\bar{\lambda}$ and exponential claims with parameter $\bar{\beta}$, fitting first three moments. Here, the idea is the same. First, we compute

$$
\bar{\beta}=\frac{3 \mu^{(2)}}{\mu^{(3)}}, \quad \bar{\lambda}=\frac{9 \lambda \mu^{(2)^{3}}}{2 \mu^{(3)^{2}}}, \quad \text { and } \quad \bar{\theta}=\frac{2 \mu \mu^{(3)}}{3 \mu^{(2)^{2}}} \theta .
$$

Next, we employ relations (1.12) and (1.13) and finally use the exact, exponential case formula (1.8) presented in Section 1.2.1. Obviously, the method gives 
Table 1.8: The finite time De Vylder approximation for for the same parameters as in Table 1.3 .

\begin{tabular}{lrrrrrr}
\hline \hline$u$ & 0 & 1 & 5 & 10 & 20 & 50 \\
\hline$\psi(u, 1)$ & 0.653971 & 0.563171 & 0.301532 & 0.130580 & 0.020847 & 0.000033 \\
$\psi(u, 2)$ & 0.720692 & 0.644069 & 0.409016 & 0.229379 & 0.069275 & 0.001321 \\
$\psi(u, 3)$ & 0.729778 & 0.655428 & 0.426237 & 0.248602 & 0.084106 & 0.003036 \\
$\psi(u, 4)$ & 0.730717 & 0.656609 & 0.428081 & 0.250765 & 0.086020 & 0.003486 \\
$\psi(u, 5)$ & 0.730769 & 0.656675 & 0.428185 & 0.250889 & 0.086136 & 0.003486 \\
\hline \hline
\end{tabular}

STF2ruin08.m

the exact result in the exponential case. For other claim distributions, the first three moments have to exist in order to apply the approximation.

Table 1.8 shows the results of the finite time De Vylder approximation with respect to the initial capital $u$ and the time horizon $T$. We see that this approximation gives even better results than the corrected diffusion approximation, compare with the reference values presented in Table 1.3.

Table 1.9 shows which approximation can be used for each claim size distribution. Moreover, the necessary assumptions on the distribution parameters are presented.

Table 1.9: Approximations and their range of applicability

\begin{tabular}{lccccccc}
\hline \hline $\begin{array}{c}\text { Distrib. } \\
\text { Method }\end{array}$ & Exp. & Gamma & $\begin{array}{c}\text { Wei- } \\
\text { bull }\end{array}$ & $\begin{array}{c}\text { Mix. } \\
\text { Exp. }\end{array}$ & $\begin{array}{c}\text { Log- } \\
\text { normal }\end{array}$ & Pareto & Burr \\
\hline Monte Carlo & + & + & + & + & + & + & + \\
Segerdahl & + & + & - & + & - & - & - \\
Brown. diff. & + & + & + & + & + & $\alpha>2$ & $\alpha \tau>2$ \\
Corr. diff. & + & + & - & + & - & - & - \\
Stable diff. & - & - & - & - & - & $\alpha<2$ & $\alpha \tau<2$ \\
Fin. De Vylder & + & + & + & + & + & $\alpha>3$ & $\alpha \tau>3$ \\
\hline \hline
\end{tabular}



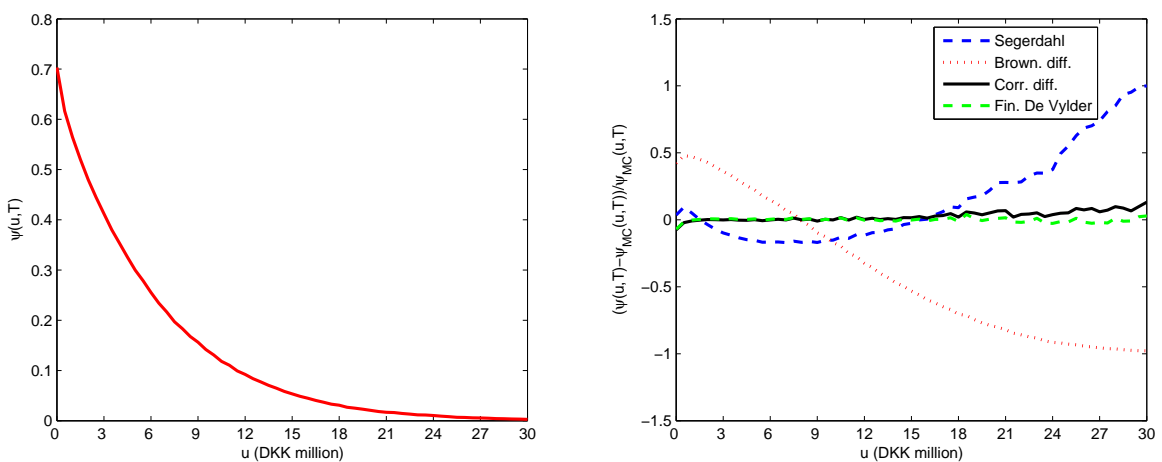

Figure 1.2: The reference ruin probability obtained via Monte Carlo simulations (left panel), the relative error of the approximations (right panel). The mixture of two exponentials case with $T$ fixed and $u$ varying.

STF2ruin09.m

\subsection{Numerical comparison of the finite time approximations}

Now, we will illustrate all six approximations presented in Section 1.3 . We consider three claim amount distributions which were best fitted to the Danish fire losses data in Chapter ??, namely the mixture of two exponentials (a running example in Section [1.3), log-normal and Pareto distributions. The parameters of the distributions are: $\beta_{1}=3.8617 \cdot 10^{-7}, \beta_{2}=3.6909 \cdot 10^{-6}$, $a=0.2568$ (mixture), $\mu=12.5247, \sigma^{2}=1.5384$ (log-normal), and $\alpha=1.3127$, $\lambda=4.0588 \cdot 10^{5}$ (Pareto). The ruin probability will be depicted as a function of $u$, ranging from 0 to 30 million DKK, with fixed $T=1$ or with fixed value of $u=5$ million DKK and varying $T$ from 0 to 5 years. The relative safety loading is set to $30 \%$. All figures have the same form of output. In the left panel, the reference ruin probability values obtained via 10 x 10000 Monte Carlo simulations are presented. The right panel depicts the relative error with respect to the reference values.

First, we consider the mixture of two exponentials. As we can see in Figures 1.2 

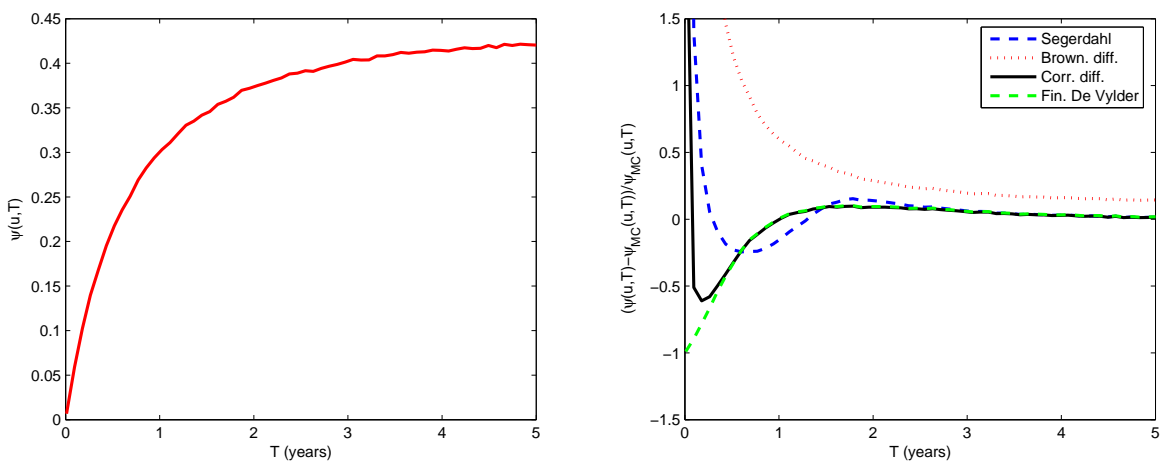

Figure 1.3: The reference ruin probability obtained via Monte Carlo simulations (left panel), the relative error of the approximations (right panel). The mixture of two exponentials case with $u$ fixed and $T$ varying.

STF2ruin10.m

and 1.3 the Brownian motion diffusion and Segerdahl approximations almost for all values of $u$ and $T$ give highly incorrect results. Corrected diffusion and finite time De Vylder approximations yield acceptable errors, which are generally below $10 \%$.

In the case of log-normally distributed claims, we can only apply two approximations: diffusion by Brownian motion and finite time De Vylder, see Table 1.9. Figures 1.4 and 1.5 depict the reference ruin probability values obtained via Monte Carlo simulations and the relative errors with respect to the reference values. Again, the finite time De Vylder approximation works better than the diffusion approximation, but, in general, the errors are not acceptable.

Finally, we take into consideration the Pareto claim size distribution. Figures 1.6 and 1.7 depict the reference ruin probability values and the relative errors with respect to the reference values for the $\alpha$-stable Lévy motion diffusion approximation (we cannot apply finite time De Vylder approximation as $\alpha<3)$. We see that the error is quite low for high values of $T$. 

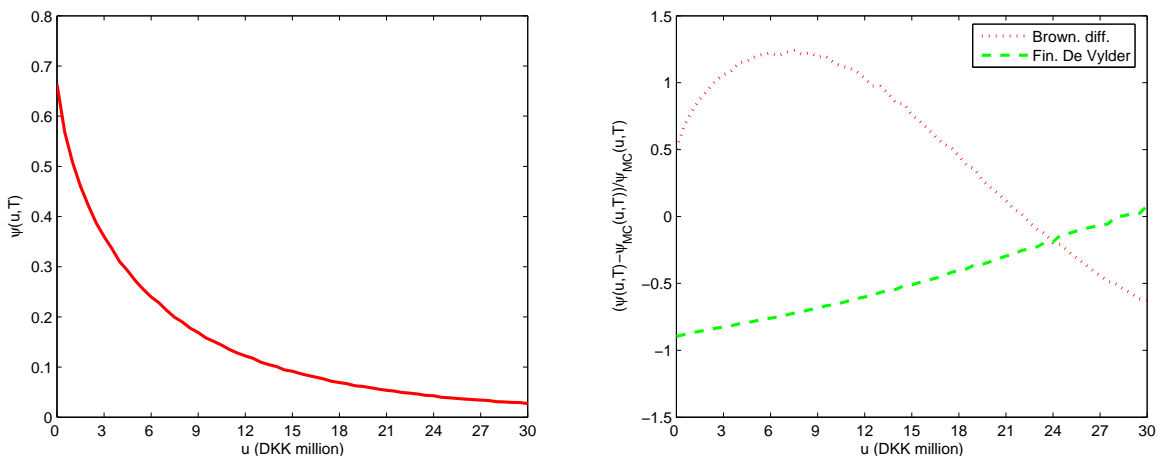

Figure 1.4: The reference ruin probability obtained via Monte Carlo simulations (left panel), the relative error of the approximations (right panel). The log-normal case with $T$ fixed and $u$ varying.

STF2ruin11.m
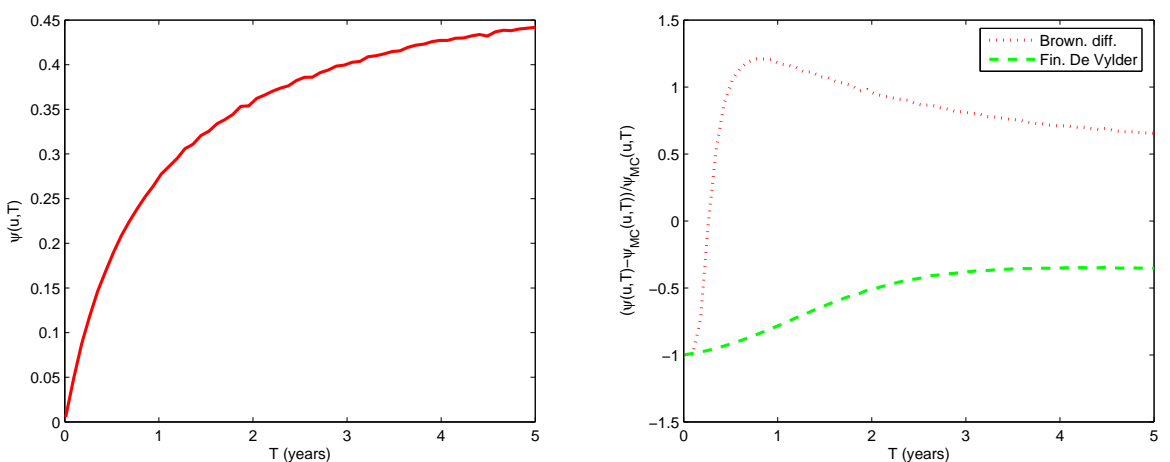

Figure 1.5: The reference ruin probability obtained via Monte Carlo simulations (left panel), the relative error of the approximations (right panel). The log-normal case with $u$ fixed and $T$ varying.

STF2ruin12.m 

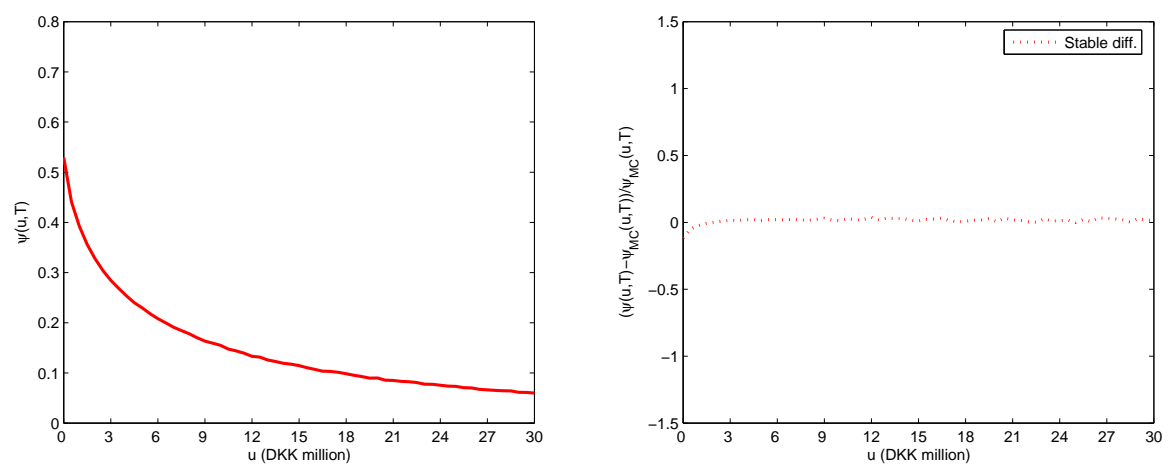

Figure 1.6: The reference ruin probability obtained via Monte Carlo simulations (left panel), the relative error of the approximation with $\alpha$ stable Lévy motion (right panel). The Pareto case with $T$ fixed and $u$ varying.

STF2ruin13.m
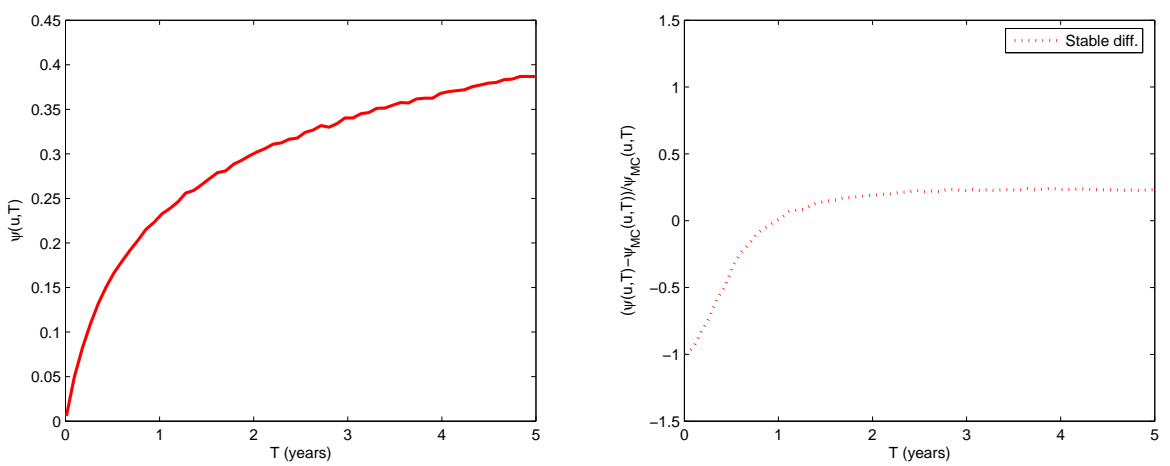

Figure 1.7: The reference ruin probability obtained via Monte Carlo simulations (left panel), the relative error of the approximation with $\alpha$ stable Lévy motion (right panel). The Pareto case with $u$ fixed and $T$ varying. 


\section{Bibliography}

Asmussen, S. (2000). Ruin Probabilities, World Scientific, Singapore.

Burnecki, K. (2000). Self-similar processes as weak limits of a risk reserve process, Probab. Math. Statist. 20(2): 261-272.

Burnecki, K., Miśta, P., and Weron, A. (2005). Ruin Probabilities in Finite and Infinite Time, in P. Cizek, W. Härdle, and R. Weron (eds.) Statistical Tools for Finance and Insurance, Springer, Berlin, 341-379.

Burnecki, K., Miśta, P., and Weron, A. (2005). What is the best approximation of ruin probability in infinite time?, Appl. Math. (Warsaw) 32(2): 155176.

Degen, M., Embrechts, P., and Lambrigger, D.D. (2007). The quantitative modeling of operational risk: between g-and-h and EVT, Astin Bulletin 37(2): 265-291.

Embrechts, P., Kaufmann, R., and Samorodnitsky, G. (2004). Ruin Theory Revisited: Stochastic Models for Operational Risk, in C. Bernadell et al (eds.) Risk Management for Central Bank Foreign Reserves, European Central Bank, Frankfurt a.M., 243-261.

Furrer, H., Michna, Z., and Weron, A. (1997). Stable Lévy motion approximation in collective risk theory, Insurance Math. Econom. 20: 97-114.

Grandell, J. (1991). Aspects of Risk Theory, Springer, New York.

Iglehart, D. L. (1969). Diffusion approximations in collective risk theory, Journal of Applied Probability 6: 285-292.

Kaishev, V.K., Dimitrova, D.S. and Ignatov, Z.G. (2008). Operational risk and insurance: a ruin-probabilistic reserving approach, Journal of Operational Risk 3(3): 39-60. 
Nolan, J.P. (2010). Stable Distributions - Models for Heavy Tailed Data, Birkhauser, Boston, In progress, Chapter 1 online at academic2.american.edu/ jpnolan.

Panjer, H.H. and Willmot, G.E. (1992). Insurance Risk Models, Society of Actuaries, Schaumburg.

Segerdahl, C.-O. (1955). When does ruin occur in the collective theory of risk?, Skand. Aktuar. Tidskr 38: 22-36. 


\section{HSC Research Report Series 2010}

For a complete list please visit http://ideas.repec.org/s/wuu/wpaper.html

01 Models for Heavy-tailed Asset Returns by Szymon Borak, Adam Misiorek and Rafał Weron

02 FX Smile in the Heston Model by Agnieszka Janek, Tino Kluge, Rafał Weron and Uwe Wystup

03 Building Loss Models by Krzysztof Burnecki, Joanna Janczura and Rafał Weron

04 Ruin Probability in Finite Time by Krzysztof Burnecki and Marek Teuerle 